\title{
Investigation of the energy deposit of inclined muon bundles in the Cherenkov water detector NEVOD
}

\author{
I.I. Yashin ${ }^{1, a}$, N.S. Barbashina ${ }^{a}$, A.G. Bogdanova , D.V. Chernov ${ }^{a}$, L.I. Dushkin ${ }^{a}$, \\ S.S. Khokhlov ${ }^{a}$, V.A. Khomyakov ${ }^{a}$, V.V. Kindin ${ }^{\text {a }}$, R.P. Kokoulin ${ }^{\text {a }}$, K.G. Kompaniets ${ }^{\text {a }}$, \\ E.A. Kovylyaeva ${ }^{a}$, G. Mannocchi ${ }^{\text {b }}$, A.A. Petrukhin ${ }^{a}$, E.V. Romanenkova ${ }^{a}$, \\ O. Saavedra ${ }^{c}$, V.V. Shutenko ${ }^{a}$, G. Trinchero ${ }^{b}$ \\ ${ }^{a}$ National Research Nuclear University MEPhI (Moscow Engineering Physics Institute), \\ 115409 Moscow, Russian Federation \\ ${ }^{b}$ Istituto di Fisica dello Spazio Interplanetario - INAF, 10133 Torino, Italy \\ ' Dipartimento di Fisica dell' Universita di Torino e INFN, 10125 Torino, Italy \\ E-mail: IIYashin@mephi.ru
}

\begin{abstract}
An excess of multi-muon events in comparison with simulations performed in frame of widely used hadron interaction models was found in several cosmic ray experiments at very- and ultrahigh energies of primary particles. In order to solve this so-called 'muon puzzle', investigations of the energy characteristics of EAS muon component are required. A possible approach to such investigations is the measurement of the energy deposit of EAS muons in the detector material; the appearance of an excessive fraction of very high-energy muons should be reflected in the dependence of the energy deposit on the energy of primary particles. The experiment on the study of the energy deposit of muon bundles is being conducted at the Experimental complex NEVOD. As a measure of the energy deposit, the sum of the responses of quasi-spherical modules of the Cherenkov water calorimeter is used. The local muon density in the event and the muon bundle arrival direction are estimated from the data of coordinate-tracking detector DECOR. Registration of inclined muon bundles of different multiplicities at various zenith angles allows to evaluate primary particle energies and to explore the energy interval from about $10^{16}$ to $10^{18} \mathrm{eV}$. Experimental data accumulated from May 2012 to April 2015 have been analyzed and compared with CORSIKA-based simulations. The average specific energy deposit (i.e., the calorimeter response normalized to the local muon density in the events) increases with zenith angle, thus reflecting the increase of the muon energy in the bundles near horizon. An evidence for an increase of the energy deposit at primary energies above $10^{17} \mathrm{eV}$ is found in the measured dependence of the specific energy deposit on the muon density. Possible reasons of such anomalous behavior are discussed.
\end{abstract}

The 34th International Cosmic Ray Conference

30 July - 6 August, 2015

The Hague, The Netherlands

\footnotetext{
${ }^{1}$ Speaker 


\section{Introduction}

In several cosmic ray experiments conducted at very- and ultra-high energies of primary particles, an excess of multi-muon events (the intensity of muon bundles, muon content in EAS) in comparison with simulations performed with widely used hadron interaction models has been found [1 - 4]. In principle, this excess may be caused as by cosmophysical (increasing abundance of heavy primary nuclei with the increase of energy) so by nuclear physical reasons (changes of characteristics of interaction of primary protons and nuclei with nuclei of air atoms). However, at energies of the order of $10^{18} \mathrm{eV}$ and higher, the excess of multi-muon events does not find its explanation in frame of the existing interaction models even under the assumption of extremely heavy (pure iron nuclei) primary composition [3 - 5]. Therefore, it seems likely that the contemporary models of the development of the nuclear cascade in the atmosphere need a revision. A key to the solution of this problem, often referred to as the "muon puzzle", may give investigations of the energy characteristics of the EAS muon component and of their changes with the primary energy [6].

One of the possible approaches to such studies is the measurement of the energy deposit of muon bundles at their passage through the detector material. The specific energy loss of muons in matter almost linearly depends on the muon energy, and the appearance of an excessive flux of high-energy muons in the bundles should lead to the change of the dependence of the average energy deposit on the primary energy. The experiment on the study of the energy deposit of the muon bundles was started at the NEVOD experimental complex in 2012. The complex includes a large volume Cherenkov water calorimeter [7, 8] and the coordinate-tracking detector [9] that provides a reliable identification of multi-muon events. Detection of muon bundles of various multiplicities in a wide range of zenith angles gives the possibility to explore a wide interval of primary particle energies in frame of a single experiment. The results of the measurements of the average energy deposit of inclined muon bundles registered during more than 17 thousand hours of obseravations and their comparison with the expected energy characteristics of muons estimated on the basis of the CORSIKA code [10] are presented below. In comparison with our preliminary analysis [11], experimental statistics has been increased by more than 5 times.

\section{Experimental data}

The measuring system of the Cherenkov water detector (CWD) NEVOD with the inner volume $9 \times 9 \times 26 \mathrm{~m}^{3}$ represents a spatial lattice of quasi-spherical modules (QSMs) [7]. Each QSM includes six FEU-200 PMTs with flat $15 \mathrm{~cm}$ diameter photocathodes directed along the axes of the orthogonal coordinate system. The measuring system contains 91 QSMs (546 PMTs) arranged in vertical strings ( 3 or 4 QSMs) with the distances $2.5 \mathrm{~m}$ along the water tank and 2.0 $m$ across it and over the depth. After a recent modernization [8], the electronic system provides the measurements of PMT signals in a wide dynamic range (from 1 to $10^{5}$ photoelectrons, ph.e.) for each PMT, thus ensuring possibilities of calorimetric studies of high energy cascade showers and muon bundles. The coordinate-tracking detector DECOR [9] with a total area about $70 \mathrm{~m}^{2}$ was specially designed for investigations of multi-particle events at large zenith angles. It includes 8 supermodules (SMs) deployed in the galleries of the NEVOD building from three sides of the water tank. The sensitive area of each SM is $8.4 \mathrm{~m}^{2}$. The supermodules consist of 8 
vertical planes of plastic streamer tube chambers and ensure the spatial and angular accuracy of muon track localization better than $1 \mathrm{~cm}$ and $1^{\circ}$, respectively. An example of the event with the muon bundle detected in the NEVOD-DECOR setup is presented in Fig. 1.

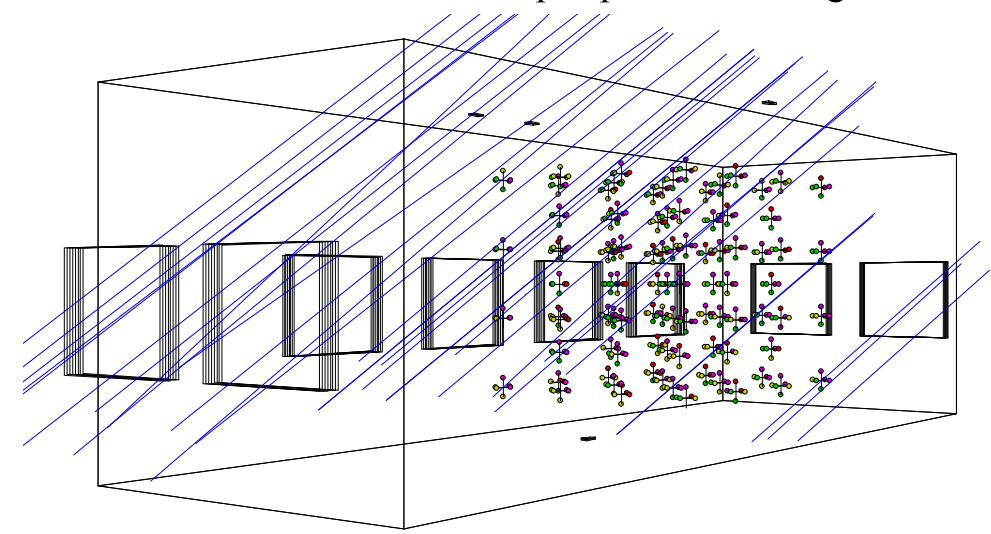

Figure 1: An example of the event with the muon bundle in NEVOD-DECOR setup. Thin lines are reconstructed muon tracks. Small circles represent hit PMTs of the CWD (colors reflect the amplitudes); big rectangles around the CWD are supermodules of the coordinate detector; small rectangles at the top and the bottom: hit scintillation counters of the calibration telescope system.

In the present analysis, data of two long-term measurement series are used: from May 2012 to March 2013 and from July 2013 to April 2015. Total live observation time is equal to $17439 \mathrm{~h}$. In these data, 29335 events with muon bundles with muon multiplicity $m \geq 5$ and zenith angles $\theta \geq 55^{\circ}$ were found. The events were selected in two $60^{\circ}$-wide sectors of azimuth angle where most of DECOR SMs (six of eight) were screened with the NEVOD water tank; data of these six shielded supermodules were used for muon track counting. Average threshold muon energy for such selection criteria is close to $2 \mathrm{GeV}$. Additionally, from the initial part of the experimental material (for $3253 \mathrm{~h}$ ) muon bundles arriving at lower zenith angles were selected $\left(40^{\circ} \leq \theta<55^{\circ}, 15084\right.$ events $)$.

As a measure of the energy deposit of the muon bundle, we use the total signal $\Sigma$ of all hit PMTs of the water calorimeter (in units of photoelectrons, ph.e.). At that, an assumption is made that the total yield of Cherenkov photons is proportional to the total muon energy loss in the detector material (including secondary particles and cascades from them). The local muon density in the event was estimated from the muon multiplicity measured in the coordinate detector taking into account the effective area $S_{\text {det }}$ of six DECOR SMs for a given direction of muon bundle arrival. It should be noted however that a straightforward estimate of the muon density as $D=m / S_{\text {det }}$ would be biased because of statistical fluctuations of the number of muons that hit the detector in conjunction with a steep spectrum of the events in the local muon density: $d F / d D=A \times D^{-(\beta+1)}$ where $\beta \sim 2.1$ is the index of the integral spectrum of muon density in the considered range [3]. Assuming that the number of muons registered in the detector at a fixed density $D$ obeys Poisson distribution with the mean value $<m>=D \times S_{\text {det }}$, the average muon density in the events which give contribution to the formation of the events with a fixed measured multiplicity $m$ may be found analytically (see section 4 of paper [3]):

$$
<D>=(m-\beta) / S_{\operatorname{det}} .
$$

In the further analysis, we use the estimate of muon density defined by this equation. 


\section{Results and discussion}

Correlations of the measured energy deposit with the local muon density estimate for a sample of events with zenith angles $\theta \geq 60^{\circ}$ are presented in Fig. 2. In the first approximation, the total energy deposit in the CWD is nearly proportional to the muon density (the solid red line in the figure), therefore further we analyze the specific energy deposit $\Sigma / D$, that is, the CWD response normalized to the muon density in the event.

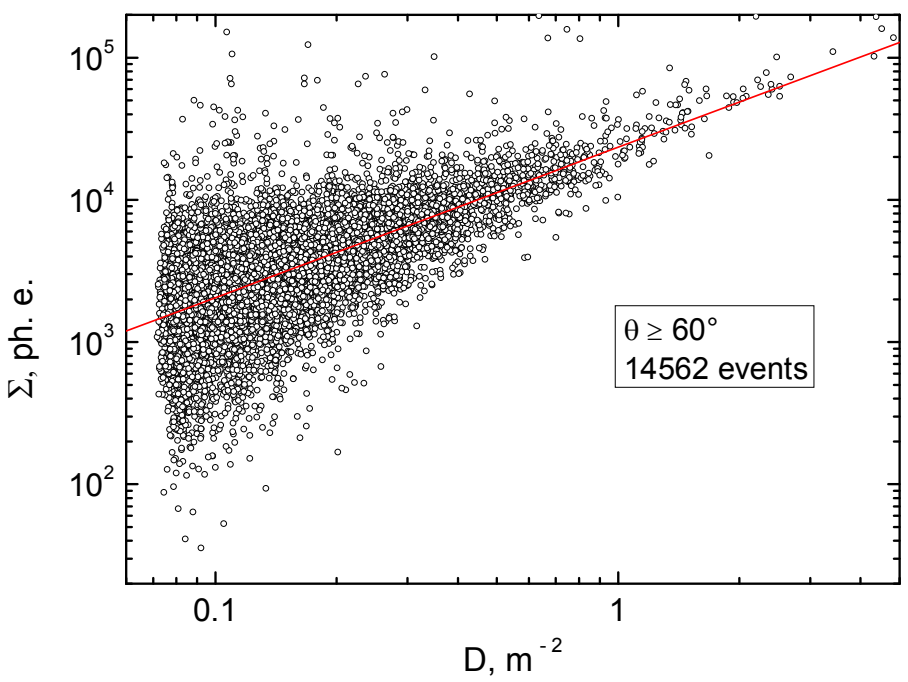

Figure 2: Correlations of the measured energy deposit and muon density estimate.

The results of the measurements of the dependence of the average specific energy deposit on the zenith angle are presented by the points in Fig. 3. The arrows in the bottom part of the figure indicate typical energies (calculated mean-logarithmic values [3]) of primary cosmic ray particles that contribute to the formation of muon bundles at different zenith angles.

At moderate zenith angles $\left(\theta<55^{\circ}\right)$, a rapid decrease of the measured energy deposit with the zenith angle increase is seen. Such dependence may be explained as a decreasing residual contribution of electron-photon and hadron components to the response of an unscreened detector deployed at the Earth surface. In this angular range, the measured dependence is well described by a negative exponent of the slant depth of the atmosphere (the dashed curve in the figure):

$$
<\Sigma / D>\sim \exp \left(-X_{0} \sec \theta / \Lambda\right)+\text { const },
$$

where $X_{0}=1014 \mathrm{~g} \mathrm{~cm}^{-2}$ is the average thickness of the atmospheric layer above the setup in the vertical direction, and $\Lambda=(134 \pm 15) \mathrm{g} \mathrm{cm}^{-2}$ is the attenuation length; the latter value is close to the absorption length of cosmic ray nucleon component in the Earth atmosphere.

At detection of muon bundles with zenith angles more than $55^{\circ}$, practically only muon component remains. As seen from the figure, the data exhibit the increase of the average energy deposit in this angular range thus indicating an increase of the mean muon energy in the bundles. The solid curves in Fig. 3 represent the expected angular dependence of the muon bundle energy deposit obtained on the basis of simulations of the EAS muon component with the CORSIKA code [10] for primary protons and iron nuclei. In simulations, we used the combination of hadron interaction models SIBYLL+FLUKA for hadrons with energies more than $80 \mathrm{GeV}$ and below this value, respectively. The calculated value of the mean energy of 
muons detected in the bundles rapidly increases at large zenith angles and reaches about 500 $\mathrm{GeV}$ near the horizon. The expected dependence of the average specific energy deposit of muon bundles in the CWD (solid curves in the figure) was calculated in the following way. First, for every zenith angle the average specific energy loss of muons $\langle d E / d X>$ was calculated using the energy loss tables [12]. Then, as mentioned above, we assumed that the total Cherenkov light yield is directly proportional to the muon energy loss. Finally, the absolute calibration of the calculated dependence was obtained by means of normalizing data and calculations in $55^{\circ}-65^{\circ}$ angular range. As a whole, the measured dependence of the average specific energy deposit on the zenith angle confirms the increase of the mean muon energy in the bundles and is in a good agreement with expectation (with a possible exception of the point between $70^{\circ}$ and $75^{\circ}$, which corresponds to effective energies of primary particles of the order of $10^{17} \mathrm{eV}$ ).

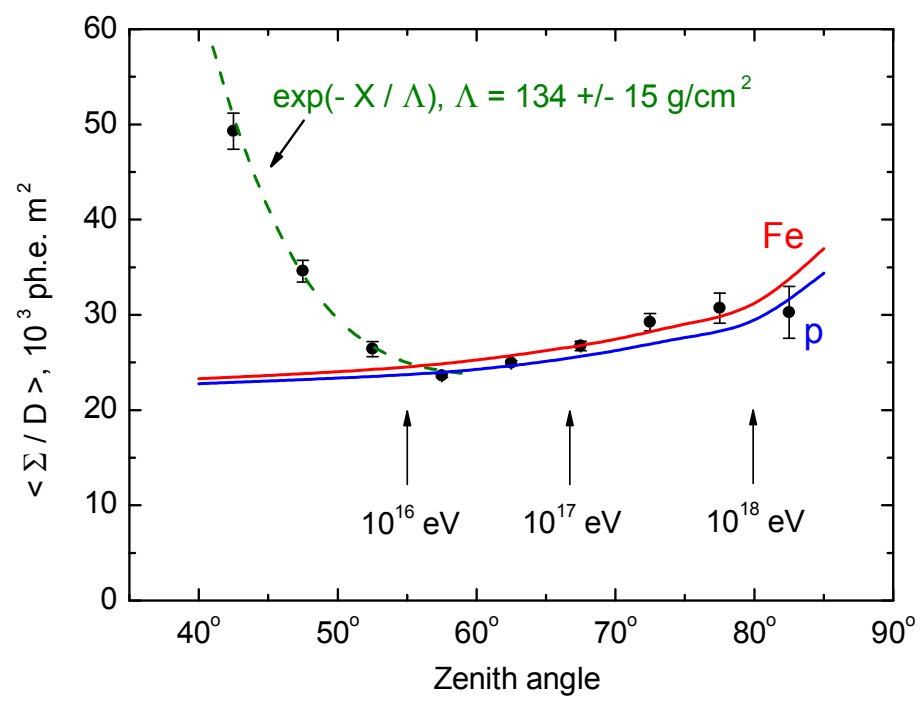

Figure 3: Dependence of the average specific energy deposit of muon bundles in the Cherenkov water detector on zenith angle. Points: results of measurement; dashed curve is the exponential fit (2) of the data in the range of zenith angles $\theta<55^{\circ}$; solid curves represent the expected dependence of muon bundle energy deposit for primary protons and iron nuclei, obtained on the basis of simulations with CORSIKA.

In order to check whether the measured zenith angular dependence of the energy deposit could be imitated by the properties of the detecting system of CWD, we have analyzed the average specific energy deposit of muon bundles as a function of the azimuth angle between the muon bundle arrival direction and the main axis of the NEVOD water reservoir (Fig. 4). As it is seen from the figure, the data demonstrate a very good uniformity of the response for various azimuth angles. Consequently, we can conclude that a non-ideally isotropic structure of the spatial lattice of quasi-spherical modules of the CWD does not distort the results of the measurements of the angular dependence of the muon bundle energy deposit.

In Fig. 5, the experimental values of the average specific CVD response for muon bundles are presented as a function of the local muon density. Data include all selected events with zenith angles more than $55^{\circ}$ where the residual contribution of electron-photon and hadron components is low. In fact, such an analysis for a fixed zenith angle interval allows to follow possible changes in the detector response (and correspondingly, in the mean muon energy in the bundles) for different primary energies. Arrows in the bottom part of the figure indicate typical (mean logarithmic) energies of primary particles which give the contribution to the formation of 
the events with corresponding values of muon density. The curves in the figure represent the expected dependence of the energy deposit on the local muon density, calculated on the basis of simulations with CORSIKA for $60^{\circ}$ zenith angle. The same calibration coefficient as in Fig. 3 was applied in order to normalize calculated results to the data.

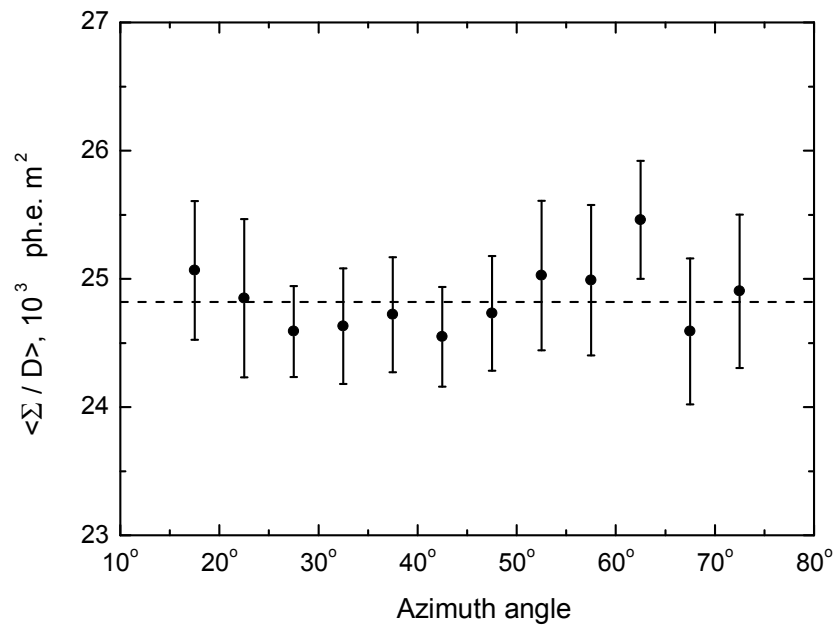

Figure 4: The dependence of the measured average specific energy deposit of muon bundles on the azimuth angle (all events with zenith angles more than $55^{\circ}$ ). The dashed line: weighted mean value.

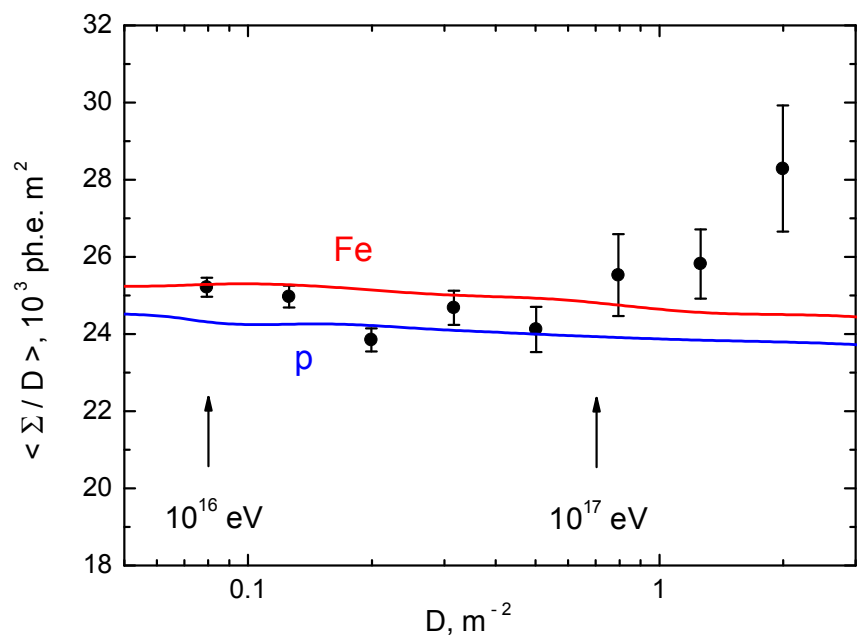

Figure 5: Dependence of the average specific energy deposit on the local muon density. Curves: calculations on the basis of the CORSIKA code for primary protons and iron nuclei.

Results of simulations demonstrate a trend to a decrease of the mean muon energy in the bundles with the increase of the primary energy. Probably, this effect is related with a logarithmic shift of the maximum of the hadronic cascade to a deeper atmosphere, and hence a decrease of the probability of decays of high-energy parent mesons. On the contrary, the data exhibit some increase of the average specific energy deposit in the region of primary energies above $10^{17} \mathrm{eV}$. The value of the increase is greater than the difference of calculated results for two limiting assumptions about the mass composition of primary particles (pure protons and pure iron nuclei), therefore this increase cannot be explained in frame of a changing primary composition and a usual hadron interaction model. Certainly, a further accumulation of statistics and a careful analysis of possible systematic effects are necessary. If confirmed, a found anomaly will represent the good evidence in favor of the inclusion of a new mechanism of 
generation of high-energy muons in interactions of hadrons and nuclei with nuclei of atmospheric atoms at ultra-high energies.

\section{Conclusion}

The experiment on the investigation of the energy characteristics of inclined muon bundles formed as a result of interactions of primary cosmic ray particles with energies $10^{16}-10^{18} \mathrm{eV}$ is being conducted at the Experimental Complex NEVOD. The aim of the experiment is the search of possible reasons of the appearance of the excessive flux of multi-muon events in ultra-high energy cosmic rays. The first results of the measurements of zenith-angular dependence of the average specific energy deposit in the Cherenkov water detector are in a reasonable agreement with CORSIKA-based simulations of the EAS muon component and confirm the increase of the mean energy of muons in the bundles at large zenith angles. An indication for an increase of the average specific energy deposit compared to the expectation at primary energies above $10^{17} \mathrm{eV}$ has been found; however, a further increase of experimental statistics and a careful analysis of possible systematic effects are necessary. If confirmed, this deviation will evidence for the inclusion of a new mechanism of generation of high-energy muons at ultra-high energies of primary particles.

The work was performed at the Unique Scientific Facility 'Experimental Complex NEVOD' with the state support provided by the Ministry of Education and Science of the Russian Federation (project RFMEFI59114X0002, the government task and the program for Leading Scientific Schools, project NSh-4930.2014.2).

\section{References}

[1] V. Avati, et al., Astropart. Phys., 19, 513 (2003).

[2] J. Abdallah, et al., Astropart. Phys., 28, 273 (2007).

[3] A.G. Bogdanov, et al., Phys. Atom. Nucl., 73, 1852 (2010).

[4] L. Nellen (for the Pierre Auger Collaboration), J. Phys.: Conf. Ser., 409, 012107 (2013).

[5] O. Saavedra, et al., J. Phys.: Conf. Ser., 409, 012009 (2013).

[6] A.A. Petrukhin, Nucl. Instrum. Meth. Phys. Res. A, 742, 228 (2014).

[7] V.M. Aynutdinov, et al., Astrophys. Space Sci., 258, 105 (1998).

[8] S.S. Khokhlov, et al., Astrophys. Space Sci. Trans., 7, 271 (2011).

[9] N.S. Barbashina, et al., Instrum. Experim. Techniques, 43, 743 (2000).

[10] D. Heck, et al., Forschungszentrum Karlsruhe Report, FZKA 6019, Karlsruhe, 1998.

[11] I.I. Yashin, et al., 33rd ICRC, Rio de Janeiro, Brazil, 2013, paper 0728; http://www.cbpf.br/ icrc2013/papers/icrc2013-0728.pdf

[12] D.E. Groom, N.V. Mokhov, S.I. Striganov, Atomic Data and Nuclear Data Tables, 78, 183 (2001). 